\title{
Spin-free relativistic no-pair ab initio core model potentials and valence basis sets for the transition metal elements Sc to Hg. II
}

\author{
Frank Rakowitz \\ Institut für Physikalische und Theoretische Chemie, Universität Bonn,
} Wegelerstr. 12, D-53115 Bonn, Germany

Christel M. Marian ${ }^{\mathrm{a})}$

GMD-Forschungszentrum für Informationstechnik GmbH, Institut für Algorithmen und Wissenschaftliches Rechnen (SCAI), Schloß Birlinghoven, D-53754 Sankt Augustin, Germany

Luis Seijo

Departamento de Química, C-XIV, Universidad Autónoma de Madrid, E-28049 Madrid, Spain

(Received 21 July 1999; accepted 20 September 1999)

\begin{abstract}
This is the second part of a report on spin-free relativistic no-pair ab initio core model potentials for the transition elements Sc to Hg. In the first part [J. Chem. Phys. 110, 3678 (1999)], we introduced the no-pair $a b$ initio model potential method and supplied model potentials for $[\mathrm{Mg}],[\mathrm{Zn}]$, and $[\mathrm{Cd}, 4 \mathrm{f}]$ cores of first-, second-, and third-row transition metals, respectively. At the Hartree-Fock level excellent agreement between all-electron and model potential results was observed for late transition metal oxides, whereas the performance of the model potentials was slightly less satisfactory for early transition metal oxides. In this paper we will present small-core model potentials corresponding to $[\mathrm{Ne}],[\mathrm{Ar}, 3 \mathrm{~d}]$, and $[\mathrm{Kr}, 4 \mathrm{~d}, 4 \mathrm{f}]$ cores, respectively. The performance of the model potentials is tested extensively in calculations on the diatomic oxides $\mathrm{VO}, \mathrm{NbO}, \mathrm{TaO}, \mathrm{NiO}$, $\mathrm{PdO}$, and $\mathrm{PtO}$, both at the Hartree-Fock level and when electron correlation is included by means of coupled-pair functional methods. Further we investigate the requirements on valence and intermediate basis sets used to represent the exchange and no-pair operators. (C) 1999 American Institute of Physics. [S0021-9606(99)30347-0]
\end{abstract}

\section{INTRODUCTION}

This is the second part of a report on no-pair onecomponent relativistic $a b$ initio model potentials (AIMPs) and valence basis sets for the first-, second-, and third-row transition metal (TM) elements. In the first part ${ }^{1}$ we presented AIMPs with $[\mathrm{Mg}],[\mathrm{Zn}]$, and $[\mathrm{Cd}, 4 \mathrm{f}]$ cores, respectively, corresponding to valence spaces comprising the $n \mathrm{~s}$, $(n-1) \mathrm{d}$, and $(n-1) \mathrm{p}$ shells where $n$ is the principal quantum number of the outermost valence shell. In the following we shall refer to these model potentials as medium-core AIMPs. Employing these medium-core AIMPs, atomic allelectron (AE) orbital energies and radial expectation values of the valence orbitals were very well reproduced. Molecular one-component relativistic AE calculations were utilized as further benchmarks to test the performance of the AIMPs. At the Hartree-Fock (HF) level AIMP and AE results for the group 10 TM monoxides were in excellent agreement, while the performance of the AIMPs was slightly less satisfactory for the group 5 TM monoxides. In particular, the dissociation energies of $\mathrm{VO}$ and $\mathrm{TaO}$ were overestimated w. r. t. the corresponding AE values whereas bond distances and vibrational frequencies were in good accord. In these cases agreement with the AE values can be improved by including the $(n-1) \mathrm{s}$ shell in the valence space and by enhancing the representation of the exchange and no-pair model potential

\footnotetext{
a) Author to whom correspondence should be addressed. Electronic mail: christel.marian@gmd.de
}

operators. For convenience, we shall call the latter representation basis sets intermediate basis sets in the following.

In the current paper, we present the details of these new model potentials and the corresponding valence and intermediate basis sets. We analyze how an improvement of the resolution of the identity affects the spectroscopic parameters of the group 5 and 10 TM monoxides. For third-row elements we also investigate the demands on f-valence basis sets. Further, we shall discuss the performance of the smalland medium-core-valence partitionings in $\mathrm{HF}$ and electron correlation calculations. Finally, we compare our data obtained at the correlated level with other theoretical and experimental work.

\section{METHOD}

The general features of the spin-free no-pair AIMP method have already been introduced in Ref. 1. For convenience, we would like to discuss here the methods for evaluating the exchange model potential and the relativistic nopair operators in more detail. In both cases intermediate basis sets are involved.

\section{A. The exchange model potential}

In the AIMP method the exchange interaction between valence and core electrons $V_{\text {exch }}$ is approximated by the exchange model potential $V_{\text {exch }}^{\mathrm{MP}}$. The molecular $V_{\text {exch }}^{\mathrm{MP}}$ is composed of atomic HF exchange potentials 


$$
\begin{aligned}
V_{\mathrm{exch}}^{\mathrm{MP}}= & \sum_{I} \sum_{l} \sum_{m=-l}^{l} \sum_{a, b}|\operatorname{alm} ; I\rangle \\
& \times\left(S_{I}^{-1} K_{I} S_{I}^{-1}\right)_{\text {almI }, \text { blm } I}\langle\text { blm } ; I|,
\end{aligned}
$$

where $S_{I}$ is the overlap matrix and $K_{I}$ the matrix representation of the exchange potential of the core at center $I$ in the intermediate basis $\{|a l m ; I\rangle\}$. In this work two different types of intermediate basis sets are used. One of them comprises all primitives of the molecular valence basis set and will be labeled val in the following. So far, this kind of resolution of the identity has been recommended for use in AIMP calculations because one-center contributions to the HF exchange potential are represented exactly in this basis. ${ }^{2}$ In the course of this work it turned out, however, that in certain cases a more complete resolution of the identity is indicated. In order to improve the intermediate basis one might, therefore, think of employing the all-electron basis instead. In this case considerable errors in the calculation of the one-center exchange potentials are introduced. From this experience we conclude that the primitives of the molecular valence basis set should be a subset of the representation basis set. The second set, denoted by the label aug.val, therefore, starts with the valence set val but is augmented by selected primitives from the AE transition metal basis. In order to avoid singularity problems in matrix inversion operations we have chosen the augmentation functions such that their exponents are not too close to those of the basis set val. These additional primitives are tabulated in Tables XII-XIV for all TM elements. ${ }^{3}$

\section{B. The spin-free relativistic no-pair operators}

The relativistic no-pair operators which have to be considered in the spin-free no-pair AIMP method are the relativistic kinetic energy operators for the valence electron $i$

$$
E_{i}=\sqrt{p_{i}^{2}+m^{2}}
$$

and the relativistically corrected interaction between the nuclei and electron $i$

$$
\begin{aligned}
V^{\mathrm{sf}}(i)= & -A_{i}\left(V_{\text {ext }}(i)+\vec{R}_{i} V_{\text {ext }}(i) \vec{R}_{i}\right) A_{i} \\
& -W_{1}^{\mathrm{sf}}(i) E_{i} W_{1}^{\mathrm{sf}}(i)-\frac{1}{2}\left\{\left(W_{1}^{\mathrm{sf}}(i)\right)^{2}, E_{i}\right\} .
\end{aligned}
$$

Herein, $V_{\text {ext }}(i)$ describes the (nonrelativistic) Coulomb attraction between electron $i$ and all nuclei, $E_{i}$ is the kinetic energy as defined above and

$$
\begin{aligned}
\vec{R}_{i} & =\frac{\vec{p}_{i}}{E_{i}+m}, \\
A_{i} & =\sqrt{\frac{E_{i}+m}{2 E_{i}}},
\end{aligned}
$$

are factors resulting from the Douglas-Kroll transformation. According to a proposal by Hess, these factors are evaluated in momentum space employing the primitive molecular basis to resolve the identity. ${ }^{4}$ Let us consider, for instance, the calculation of the matrix element $M$ of a single one-electron term of Eq. (3)

$$
M=\left\langle a l_{a}(i)\left|A_{i} \vec{R}_{i} V_{\text {ext }}(i) \vec{R}_{i} A_{i}\right| b l_{b}(i)\right\rangle .
$$

$M$ is calculated by the insertion of several intermediate basis sets $\left\{\left|\alpha_{p}(i)\right\rangle\right\}$ and $\left\{\left|k l_{k}(i)\right\rangle\right\} .^{5}$

$$
\begin{aligned}
M= & \sum_{\alpha, \beta, \gamma, \delta, k, n}\left\langle a l_{a}(i) \mid \alpha_{p}(i)\right\rangle\left\langle\alpha_{p}(i)\left|\frac{A_{i}}{E_{i}+m}\right| \beta_{p}(i)\right\rangle \\
& \times\left\langle\beta_{p}(i) \mid k l_{k}(i)\right\rangle\left\langle k l_{k}(i)\right|\left(-i \nabla_{i}\right) V_{\mathrm{ext}}(i) \\
& \times\left(-i \nabla_{i}\right)\left|n l_{n}(i)\right\rangle \\
& \times\left\langle n l_{n}(i) \mid \gamma_{p}(i)\right\rangle\left\langle\gamma_{p}(i)\left|\frac{A_{i}}{E_{i}+m}\right| \delta_{p}(i)\right\rangle \\
& \times\left\langle\delta_{p}(i) \mid b l_{b}(i)\right\rangle
\end{aligned}
$$

where the $\left\{\alpha_{p}(i)\right\}$ are functions of momentum space and $\left\{k l_{k}(i)\right\}$ is the intermediate basis defined in ordinary space. The basis functions $\left\{\alpha_{p}(i)\right\}$ are obtained by diagonalizing the matrix of the nonrelativistic kinetic energy $p^{2} / 2 m$ represented in the original intermediate basis set. Note that integrals of type $\left\langle a l_{a}(i) \mid \alpha_{p}(i)\right\rangle$ are not just simple overlap integrals, but represent Fourier transforms between real and momentum space. As for the exchange model potential, we will use intermediate basis sets of types val and aug.val, respectively.

\section{MODEL POTENTIALS, BASIS SETS, AND ATOMIC RESULTS}

In this section we present model potentials and valence basis sets for the TMs which we will refer to as small-core AIMPs. They correspond to the $[\mathrm{Ne}],[\mathrm{Ar}, 3 \mathrm{~d}]$, and $[\mathrm{Kr}$, $4 \mathrm{~d}, 4 \mathrm{f}]$ cores, respectively. The exponents of the primitive Gaussian functions used to describe the $n \mathrm{~s},(n-1) \mathrm{d},(n$ $-1) \mathrm{p}$, and $(n-1) \mathrm{s}$ valence shells are the same as in Ref. 1 . Atomic AIMP calculations were carried out with a modified MOLECULE-SWEDEN $^{6}$ package and the ECPAIMP ${ }^{7}$ code. We have determined contraction coefficients in atomic relativistic no-pair CASSCF (complete active space self-consistent field) calculations with $x+2$ active electrons in the active $n \mathrm{~s}$ and $(n-1) \mathrm{d}$ shells where $x$ denotes the $\mathrm{d}$ shell occupation in an atomic configuration with a closed valence s shell. The actual atomic configurations and the newly optimized coefficients are presented together with the corresponding exponents in Tables IX-XI. ${ }^{3}$ The level shifters can be constructed from the data already presented in Ref. 1 by removing the part corresponding to the $(n-1) \mathrm{s}$ shell from the AIMP Hamiltonian. The small-core Coulomb model potentials for the TMs are displayed in Tables VI-VIII. ${ }^{3}$ The small-core AIMP valence orbital energies and radial expectation values show the same good quality and agreement with AE results as we already observed for the medium-core AIMPs.

\section{MOLECULAR CALCULATIONS}

Molecular calculations are performed for the group 5 and 10 TM monoxides at the AE and AIMP levels employing the MOLCAS ${ }^{8}$ package. Transition metal AE basis sets, the medium-core TM AIMP basis sets, and the oxygen basis set have already been described in Ref. 1 . The same polarization functions were used to augment the medium-core and small- 
TABLE I. Comparison of spectroscopic parameters obtained from AE, medium-core and small-core AIMP calculations at the Hartree-Fock level (if not noted otherwise). The labels val and aug.val denote different intermediate basis sets (see text).

\begin{tabular}{|c|c|c|c|c|c|c|c|c|}
\hline \multirow[b]{2}{*}{ Molecule } & \multirow[b]{2}{*}{ State } & & \multirow[b]{2}{*}{ Core } & \multicolumn{2}{|c|}{ Representation } & \multirow[b]{2}{*}{$R_{e}[\AA]$} & \multirow[b]{2}{*}{$\omega\left[\mathrm{cm}^{-1}\right]$} & \multirow[b]{2}{*}{$D_{e}[\mathrm{eV}$} \\
\hline & & & & Exchange & Relativistic & & & \\
\hline \multirow[t]{5}{*}{ VO } & ${ }^{4} \Sigma^{-}$ & AIMP & {$[\mathrm{Mg}]$} & val & val & 1.542 & 1199 & 1.72 \\
\hline & & AIMP & {$[\mathrm{Mg}]$} & aug.val & aug.val & 1.545 & 1187 & 1.63 \\
\hline & & AIMP & {$[\mathrm{Ne}]$} & val & val & 1.551 & 1191 & 1.59 \\
\hline & & AIMP & {$[\mathrm{Ne}]$} & aug.val & aug.val & 1.552 & 1185 & 1.54 \\
\hline & & $\mathrm{AE}$ & $\cdots$ & $\cdots$ & $\mathrm{ae}$ & 1.555 & 1178 & 1.53 \\
\hline \multirow[t]{5}{*}{$\mathrm{NbO}$} & ${ }^{4} \Sigma^{-}$ & AIMP & {$[\mathrm{Zn}]$} & val & val & 1.657 & 1080 & 3.46 \\
\hline & & AIMP & {$[\mathrm{Zn}]$} & aug.val & aug.val & 1.655 & 1099 & 3.59 \\
\hline & & AIMP & {$[\mathrm{Ar}, 3 \mathrm{~d}]$} & val & val & 1.665 & 1089 & 3.35 \\
\hline & & AIMP & {$[\mathrm{Ar}, 3 \mathrm{~d}]$} & aug.val & aug.val & 1.661 & 1110 & 3.53 \\
\hline & & $\mathrm{AE}$ & $\cdots$ & $\cdots$ & ae & 1.658 & 1105 & 3.51 \\
\hline \multirow[t]{5}{*}{$\mathrm{TaO}$} & ${ }^{4} \Sigma^{-}$ & AIMP & {$[\mathrm{Cd}, 4 \mathrm{f}]$} & val & val & 1.683 & 1068 & 4.42 \\
\hline & & AIMP & {$[\mathrm{Cd}, 4 \mathrm{f}]$} & aug.val & aug.val & 1.686 & 1061 & 4.33 \\
\hline & & AIMP & {$[\mathrm{Kr}, 4 \mathrm{~d}, 4 \mathrm{f}]$} & val & val & 1.686 & 1074 & 4.36 \\
\hline & & AIMP & {$[\mathrm{Kr}, 4 \mathrm{~d}, 4 \mathrm{f}]$} & aug.val & aug.val & 1.689 & 1066 & 4.26 \\
\hline & & $\mathrm{AE}$ & $\cdots$ & $\cdots$ & ae & 1.693 & 1070 & 4.21 \\
\hline \multirow[t]{5}{*}{$\mathrm{NiO}$} & ${ }^{3} \Sigma^{-}$ & AIMP & {$[\mathrm{Mg}]$} & val & val & 1.673 & 769 & -1.65 \\
\hline & & AIMP & {$[\mathrm{Mg}]$} & aug.val & aug.val & 1.675 & 771 & -1.68 \\
\hline & & AIMP & {$[\mathrm{Ne}]$} & val & val & 1.677 & 768 & -1.66 \\
\hline & & AIMP & {$[\mathrm{Ne}]$} & aug.val & aug.val & 1.678 & 769 & -1.70 \\
\hline & & $\mathrm{AE}$ & $\cdots$ & $\cdots$ & $\mathrm{ae}$ & 1.674 & 773 & -1.72 \\
\hline \multirow[t]{5}{*}{$\mathrm{PdO}$} & ${ }^{3} \Pi$ & AIMP & {$[\mathrm{Zn}]$} & val & val & 1.980 & 549 & 0.80 \\
\hline & & AIMP & {$[\mathrm{Zn}]$} & aug.val & aug.val & 1.974 & 548 & 0.81 \\
\hline & & AIMP & {$[\mathrm{Ar}, 3 \mathrm{~d}]$} & val & val & 1.987 & 547 & 0.78 \\
\hline & & AIMP & {$[\mathrm{Ar}, 3 \mathrm{~d}]$} & aug.val & aug.val & 1.977 & 548 & 0.79 \\
\hline & & $\mathrm{AE}$ & $\cdots$ & $\cdots$ & ae & 1.981 & 549 & 0.75 \\
\hline \multirow[t]{5}{*}{$\mathrm{PtO}$} & ${ }^{3} \Sigma^{-a}$ & AIMP & {$[\mathrm{Cd}, 4 \mathrm{f}]$} & val & val & 1.799 & 706 & 0.92 \\
\hline & & AIMP & {$[\mathrm{Cd}, 4 \mathrm{f}]$} & aug.val & aug.val & 1.807 & 695 & 0.87 \\
\hline & & AIMP & {$[\mathrm{Kr}, 4 \mathrm{~d}, 4 \mathrm{f}]$} & val & val & 1.801 & 701 & 0.91 \\
\hline & & AIMP & {$[\mathrm{Kr}, 4 \mathrm{~d}, 4 \mathrm{f}]$} & aug.val & aug.val & 1.812 & 685 & 0.83 \\
\hline & & $\mathrm{AE}$ & $\cdots$ & $\ldots$ & $\mathrm{ae}$ & 1.808 & 686 & 0.89 \\
\hline
\end{tabular}

${ }^{\mathrm{a}}$ CASSCF calculation distributing six electrons in the $\pi$ and $\pi^{\prime}$ orbitals.

core valence basis sets. For the first- and second-row TM elements the final contraction patterns for the small-core AIMP valence basis read $[5 \mathrm{~s} / 4 \mathrm{p} / 4 \mathrm{~d} / 1 \mathrm{f}]$, whereas for the third-row elements a $[5 \mathrm{~s} / 4 \mathrm{p} / 4 \mathrm{~d} / 2 \mathrm{f}]$ contraction is utilized. Unless noted otherwise all calculations at the correlated level were performed employing the modified coupled-pair functional (MCPF) method. ${ }^{9}$ For multi-reference cases the averaged coupled-pair functional (ACPF) approach was used. ${ }^{10}$ In either case single and double excitations from the set of $n \mathrm{~s}$ and $(n-1) \mathrm{d}$ orbitals were allowed, while restricting the replacements in the $(n-1) \mathrm{p}$ shell of the TM to a single hole.

For a comparison of AE and AIMP results the same valence electron configuration $\left(\sigma^{1} \delta^{2},{ }^{4} \Sigma^{-}\right)$was chosen for $\mathrm{VO}, \mathrm{NbO}$, and $\mathrm{TaO}$. For the lighter homologes $\mathrm{VO}$ and $\mathrm{NbO}$ this electron configuration corresponds to the ground state, ${ }^{11,12}$ whereas it yields a low-lying excited state in $\mathrm{TaO}^{13}$ Due to the relativistic inert-pair effect the heavier $\mathrm{TaO}$ prefers a $\sigma^{2} \delta^{1}$ configuration leading to a ${ }^{2} \Delta$ electronic ground state with $\Omega=3 / 2$ and $\Omega=5 / 2$ spin-orbit sublevels. In Sec. IV C, therefore, averaged spectroscopic parameters of these states are chosen as experimental reference.

The situation is different for $\mathrm{NiO}, \mathrm{PdO}$, and $\mathrm{PtO}$. All three diatomic oxides exhibit a ${ }^{3} \Sigma^{-}$ground state or finestructure components hereof. Comparison with experiment is therefore made for this state with $\sigma^{2} \pi^{2}$ configuration. In $\mathrm{PdO}$ this state is not bound at the HF level, and we thus compare AE and AIMP results for the excited $\left(\sigma^{1} \pi^{3}\right)^{3} \Pi$ state of this molecule.

\section{A. Discussion of AIMP and AE Hartree-Fock results}

In Table I we list equilibrium distances, vibrational frequencies and dissociation energies of the ground or lowlying states of the group 5 and $10 \mathrm{TM}$ monoxides. Unless stated otherwise, all calculations discussed in this section have been performed at the Hartree-Fock level.

The quality of the AIMP results can be estimated by comparison with corresponding AE values. Regardless of the core or intermediate basis set size, bond distances and vibrational frequencies are in excellent agreement with $\mathrm{AE}$ results. Concerning dissociation energies, deviations from $\mathrm{AE}$ results are within $0.2 \mathrm{eV}$, which can be accepted for any kind of effective core potential (ECP). Nevertheless some interesting trends in spectroscopic parameters are observed for the different types of AIMPs. When smaller AIMP cores are 
TABLE II. Investigation of the representation basis set completeness of the exchange model potential and relativistic no-pair operators at the Hartree-Fock level using small-core AIMPs. The different sets of calculations were performed at the same interatomic distance close to the respective equilibrium distance of each molecule. Thus the dissociation energies $D$ presented are only approximate. The labels val and aug.val denote different intermediate basis sets (see text).

\begin{tabular}{|c|c|c|c|c|c|c|c|}
\hline \multirow[b]{2}{*}{ Molecule } & \multirow[b]{2}{*}{ State } & \multirow[b]{2}{*}{ d-occupation } & \multirow[b]{2}{*}{ Core } & \multicolumn{2}{|c|}{ Representation } & \multirow{2}{*}{$\begin{array}{c}D[\mathbf{e V}] / \\
\Delta D[\mathrm{eV}]\end{array}$} & \multirow[b]{2}{*}{ TM state } \\
\hline & & & & Exchange & Relativistic & & \\
\hline \multirow[t]{3}{*}{ VO } & ${ }^{4} \Sigma^{-}$ & 3.2 & {$[\mathrm{Ne}]$} & val & val & 1.549 & ${ }^{4} \mathrm{~F}\left(\mathrm{~d}^{3} \mathrm{~s}^{2}\right)$ \\
\hline & & & & val & aug.val & +0.002 & \\
\hline & & & & aug.val & aug.val & -0.046 & \\
\hline \multirow[t]{3}{*}{$\mathrm{NbO}$} & ${ }^{4} \Sigma^{-}$ & 3.3 & {$[\mathrm{Ar}, 3 \mathrm{~d}]$} & val & val & 3.330 & ${ }^{6} \mathrm{D}\left(\mathrm{d}^{4} \mathrm{~s}^{1}\right)$ \\
\hline & & & & val & aug.val & -0.003 & \\
\hline & & & & aug.val & aug.val & +0.184 & \\
\hline \multirow[t]{3}{*}{$\mathrm{TaO}$} & ${ }^{4} \Sigma^{-}$ & 3.2 & {$[\mathrm{Kr}, 4 \mathrm{~d}, 4 \mathrm{f}]$} & val & val & 4.362 & ${ }^{4} \mathrm{~F}\left(\mathrm{~d}^{3} \mathrm{~s}^{2}\right)$ \\
\hline & & & & val & aug.val & +0.005 & \\
\hline & & & & aug.val & aug.val & -0.099 & \\
\hline \multirow[t]{3}{*}{$\mathrm{NiO}$} & ${ }^{3} \Sigma^{-}$ & 8.2 & {$[\mathrm{Ne}]$} & val & val & -1.668 & ${ }^{3} \mathrm{~F}\left(\mathrm{~d}^{8} \mathrm{~s}^{2}\right)$ \\
\hline & & & & val & aug.val & +0.004 & \\
\hline & & & & aug.val & aug.val & -0.040 & \\
\hline \multirow[t]{3}{*}{$\mathrm{PdO}$} & ${ }^{3} \Pi$ & 9.1 & {$[\mathrm{Ar}, 3 \mathrm{~d}]$} & val & val & 0.776 & ${ }^{3} \mathrm{D}\left(\mathrm{d}^{9} \mathrm{~s}^{1}\right)$ \\
\hline & & & & val & aug.val & +0.005 & \\
\hline & & & & aug.val & aug.val & +0.008 & \\
\hline \multirow[t]{3}{*}{$\mathrm{PtO}^{\mathrm{a}}$} & ${ }^{3} \Sigma^{-}$ & 8.7 & {$[\mathrm{Kr}, 4 \mathrm{~d}, 4 \mathrm{f}]$} & val & val & 0.905 & ${ }^{3} \mathrm{D}\left(\mathrm{d}^{9} \mathrm{~s}^{1}\right)$ \\
\hline & & & & val & aug.val & -0.005 & \\
\hline & & & & aug.val & aug.val & -0.072 & \\
\hline
\end{tabular}

${ }^{\mathrm{a}} \mathrm{CASSCF}$ calculation distributing six electrons in the $\pi$ and $\pi^{\prime}$ orbitals.

employed, typically equilibrium distances are increased, dissociation energies are decreased, while vibrational frequencies remain almost unchanged. As expected, the effects due to reducing the core size are more distinct for the early TM elements, because the radial extent of their cores is larger and the $(n-1) \mathrm{s}$ shells are easier polarized. Further, the dissociation energies of the early TM oxides obtained at the AIMP level appear to be sensitive to the size of intermediate basis. The deviations from the corresponding AE results are diminished when the matrix representation of the exchange and relativistic operators is improved. Excellent agreement is observed when spectroscopic parameters obtained at the $\mathrm{AE}$ level are compared with the "best" type of AIMP calculations, i.e., when small-core AIMPs and intermediate basis sets of aug.val type are used.

In order to check the quality requirements on the intermediate basis sets we carried out three series of test calculations. In all cases we employed small-core AIMPs. In the first series both the exchange model potentials and the relativistic no-pair operators were represented by intermediate basis sets of type val, in the second we used aug.val no-pair basis sets and val exchange model potential basis sets and, finally, in the third series aug.val representation basis sets were used for both types of operators. For reasons of comparability, the different sets of calculations on a specific molecule were performed at the same interatomic distance close to the respective equilibrium distance. Thus the dissociation energies presented in Table II are only approximate. The inclusion of additional basis functions for the representation of the relativistic no-pair operators do not alter the results, i.e., intermediate basis sets of type val are already approximately complete for this type of operator. A rationale of this result is the fact that the kinematic relativistic correction factors $R$ and $A$ [Eqs. (4) and (5)] affect mainly regions of high linear momentum, e.g., close to a nucleus. The most significant relativistic corrections affect the core electrons, which have already been incorporated in the model potential (MP). When the representation of the exchange model potential is improved as well (fifth entries in Table II), the changes in the dissociation energies are more distinct. This means that the val representation sets have some deficiencies in this case. Similar observations were made for nonrelativistic test calculations on first-row transition metal oxides, i.e., when the intermediate basis was used solely for resolving the identity in the exchange matrix elements. We can, therefore, safely assume that the changes in the dissociation energies mainly arise from the improved representation of the exchange model potential operator. As both types of intermediate basis sets-val and val.aug-allow for the exact calculation of one-center exchange model potential operator contributions (cf. Sec. II), the two-center exchange terms must be responsible for the observed changes. Very similar effects are found for medium-core and small-core AIMPs. We are, therefore, lead to the conclusion that the representation of the twocenter exchange interaction of the valence shells with the semicore $(n-1) \mathrm{s}$ is not critical but that rather inner core orbitals are involved.

Next, we tested the performance of different $f$ valence basis sets for the third-row transition metal elements. This question is of technical interest since the calculation of inte- 
TABLE III. Comparison of different $f$ valence basis sets (f-VBS). For all cases the AIMP calculations were performed with the same aug.val representation basis set.

\begin{tabular}{|c|c|c|c|c|c|c|c|c|c|}
\hline \multirow[b]{2}{*}{ Molecule } & \multirow[b]{2}{*}{ State } & & \multirow[b]{2}{*}{ Core } & \multicolumn{2}{|c|}{ Representation } & \multirow[b]{2}{*}{ f-VBS } & \multirow[b]{2}{*}{$R_{e}[\AA]$} & \multirow[b]{2}{*}{$\omega\left[\mathrm{cm}^{-1}\right]$} & \multirow[b]{2}{*}{$D_{e}[\mathrm{eV}]$} \\
\hline & & & & Exchange & Relativistic & & & & \\
\hline \multirow[t]{7}{*}{$\mathrm{TaO}$} & ${ }^{4} \Sigma^{-}$ & $\mathrm{AE}$ & $\ldots$ & $\ldots$ & ae & {$[9,1]$} & 1.693 & 1070 & 4.21 \\
\hline & & AIMP & {$[\mathrm{Cd}, 4 \mathrm{f}]$} & val & val & {$[5,1]$} & 1.683 & 1068 & 4.42 \\
\hline & & AIMP & {$[\mathrm{Cd}, 4 \mathrm{f}]$} & val & val & {$[1,1]$} & 1.672 & 1058 & 4.54 \\
\hline & & AIMP & {$[\mathrm{Cd}, 4 \mathrm{f}]$} & val & val & {$[-, 1]$} & 1.686 & 1078 & 4.52 \\
\hline & & AIMP & {$[\mathrm{Cd}, 4 \mathrm{f}]$} & aug.val & aug.val & {$[5,1]$} & 1.686 & 1061 & 4.33 \\
\hline & & AIMP & {$[\mathrm{Cd}, 4 \mathrm{f}]$} & aug.val & aug.val & {$[1,1]$} & 1.682 & 1059 & 4.34 \\
\hline & & AIMP & {$[\mathrm{Cd}, 4 \mathrm{f}]$} & aug.val & aug.val & {$[-, 1]$} & 1.687 & 1057 & 4.26 \\
\hline \multirow[t]{7}{*}{$\mathrm{PtO}$} & ${ }^{3} \Sigma^{-}$ & $\mathrm{AE}$ & $\cdots$ & $\cdots$ & ae & {$[9,1]$} & 1.808 & 686 & 0.89 \\
\hline & & AIMP & {$[\mathrm{Cd}, 4 \mathrm{f}]$} & val & val & {$[5,1]$} & 1.799 & 706 & 0.92 \\
\hline & & AIMP & {$[\mathrm{Cd}, 4 \mathrm{f}]$} & val & val & {$[1,1]$} & 1.799 & 714 & 0.97 \\
\hline & & AIMP & {$[\mathrm{Cd}, 4 \mathrm{f}]$} & val & val & {$[-, 1]$} & 1.793 & 720 & 0.99 \\
\hline & & AIMP & {$[\mathrm{Cd}, 4 \mathrm{f}]$} & aug.val & aug.val & {$[5,1]$} & 1.807 & 695 & 0.87 \\
\hline & & AIMP & {$[\mathrm{Cd}, 4 \mathrm{f}]$} & aug.val & aug.val & {$[1,1]$} & 1.810 & 692 & 0.84 \\
\hline & & AIMP & {$[\mathrm{Cd}, 4 \mathrm{f}]$} & aug.val & aug.val & {$[-, 1]$} & 1.812 & 691 & 0.84 \\
\hline
\end{tabular}

grals involving shells of angular momentum quantum number $l=3$ or higher are the most memory and disk consuming. The original AIMP $\mathrm{f}$ valence basis sets ${ }^{1}$ comprise one fiveprimitive contracted function plus one f polarization primitive $([5,1])$. Exponents and contraction coefficients of the former were fitted to the all-electron atomic $4 \mathrm{f}$ orbital. ${ }^{14}$ In a first step the four primitives exhibiting the largest exponents are removed from the contracted function resulting to $[1,1]$ valence basis sets. In a second step the remaining single primitive is removed as well and just a single $f$ polarization primitive remains in the valence basis set $([-, 1])$. This means that in case the valence basis (val) is employed for resolving the identity also the representation of the exchange and relativistic operators changes. By contrast, the intermediate basis remains the same in all (aug.val) calculations, i.e., in these cases always seven primitive $f$ functions represent the MP Hamiltonian. Table III displays the results of AE and medium-core AIMP calculations at the HF or CASSCF levels, respectively. The changes of the $\mathrm{TaO}$ and $\mathrm{PtO}$ spectroscopic parameters are quite small when the $f$ valence basis sets are reduced while retaining the enhanced representation basis (aug.val). In this case it appears, therefore, sufficient to keep just the $\mathrm{f}$ polarization function in the valence basis set. Since intermediate representation basis sets are involved in the calculation of one-electron integrals only the additional memory and disk requirements are negligible. In contrast, the use of reduced $f$ valence basis sets saves considerable resources in the evaluation of the two-electron integrals. We would like to note that this procedure is not recommendable, if only the valence primitives are used as intermediate basis sets. In the latter case, the reduction of the $\mathrm{f}$ basis leads to a further increase of the dissociation energies enlarging the deviation from the AE result.

\section{B. Correlation calculations}

When correlation effects are included, the spectroscopic parameters are changed significantly compared to the HF level of calculation. The corresponding spectroscopic parameters are presented in Table IV. Not surprisingly, the most distinct effect is observed for the dissociation energies which prove to be totally unrealistic at the HF level. At the correlated level the group $5 \mathrm{TM}$ monoxides exhibit dissociation energies in the range of 6 to $7 \mathrm{eV}$ while the group $10 \mathrm{TM}$ monoxides show dissociation energies of approximately half this amount only.

Comparing the results of the AIMP and AE calculations at the correlated level we find that the effects of including the $(n-1)$ s shell are slightly more pronounced than at the HF level. Equilibrium distances of the group $5 \mathrm{TM}$ monoxides increase by $\sim 0.01-0.02 \AA$ when small-core AIMPs are used instead of medium-core AIMPs. Concerning the augmentation of the intermediate basis, the same trends-both in direction and magnitude-are observed as in the HF calculations, in consistence with the fact that this augmentation improves the representation of the core exchange operator, which is an effective one-electron operator. Again, the smallcore AIMP results, applying the aug.val representation basis sets, are in excellent agreement with the AE ones. The largest deviations occur for PtO, i.e., $0.013 \AA$ in the bond length, $25 \mathrm{~cm}^{-1}$ in the harmonic vibrational frequency and $0.17 \mathrm{eV}$ in the dissociation energy, which we consider to be still very good.

\section{Comparison with other theoretical and experimental work}

In Table $\mathrm{V}$ our results at the correlated level are compared with other theoretical and experimental work. For all group 5 TM monoxides, very good to excellent agreement with experimental results is found. Compared to the theoretical studies by Bauschlicher and Langhoff ${ }^{15,16}$ and Dolg et al. ${ }^{13,17}$ results of similar or better quality are obtained in the present study. Our calculated dissociation energies retain $91 \%-95 \%$ of the experimentally determined values, vibrational frequencies and equilibrium distances are reproduced to within an error of at most $23 \mathrm{~cm}^{-1}$ or $0.015 \AA$, respectively. Part of the remaining errors are due to deficiencies in the oxygen basis which is a [4s3p2d] set of generalized contracted Gaussians. In the diatomic oxides oxygen carries a 
TABLE IV. Comparison of spectroscopic parameters obtained from AE, medium-core and small-core AIMP modified coupled-pair functional (if not noted otherwise) calculations. The labels val and aug.val denote different intermediate basis sets (see text).

\begin{tabular}{|c|c|c|c|c|c|c|c|c|}
\hline \multirow[b]{2}{*}{ Molecule } & \multirow[b]{2}{*}{ State } & & \multirow[b]{2}{*}{ Core } & \multicolumn{2}{|c|}{ Representation } & \multirow[b]{2}{*}{$R_{e}[\AA]$} & \multirow[b]{2}{*}{$\omega\left[\mathrm{cm}^{-1}\right]$} & \multirow[b]{2}{*}{$D_{e}[\mathrm{eV}]$} \\
\hline & & & & Exchange & Relativistic & & & \\
\hline \multirow[t]{5}{*}{ VO } & ${ }^{4} \Sigma^{-}$ & AIMP & {$[\mathrm{Mg}]$} & val & val & 1.565 & 1025 & 6.38 \\
\hline & & AIMP & {$[\mathrm{Mg}]$} & aug.val & aug.val & 1.568 & 1017 & 6.31 \\
\hline & & AIMP & {$[\mathrm{Ne}]$} & val & val & 1.585 & 1010 & 6.12 \\
\hline & & AIMP & {$[\mathrm{Ne}]$} & aug.val & aug.val & 1.586 & 1008 & 6.09 \\
\hline & & $\mathrm{AE}$ & $\cdots$ & $\cdots$ & $\mathrm{ae}$ & 1.588 & 1003 & 6.06 \\
\hline \multirow[t]{5}{*}{$\mathrm{NbO}$} & ${ }^{4} \Sigma^{-}$ & AIMP & {$[\mathrm{Zn}]$} & val & val & 1.665 & 1012 & 7.31 \\
\hline & & AIMP & {$[\mathrm{Zn}]$} & aug.val & aug.val & 1.662 & 1031 & 7.42 \\
\hline & & AIMP & {$[\mathrm{Ar}, 3 \mathrm{~d}]$} & val & val & 1.685 & 998 & 7.05 \\
\hline & & AIMP & {$[\mathrm{Ar}, 3 \mathrm{~d}]$} & aug.val & aug.val & 1.680 & 1022 & 7.21 \\
\hline & & $\mathrm{AE}$ & $\cdots$ & $\cdots$ & ae & 1.676 & 1022 & 7.23 \\
\hline \multirow[t]{5}{*}{$\mathrm{TaO}$} & ${ }^{4} \Sigma^{-}$ & AIMP & {$[\mathrm{Cd}, 4 \mathrm{f}]$} & val & val & 1.692 & 992 & 7.33 \\
\hline & & AIMP & {$[\mathrm{Cd}, 4 \mathrm{f}]$} & aug.val & aug.val & 1.695 & 984 & 7.25 \\
\hline & & AIMP & {$[\mathrm{Kr}, 4 \mathrm{~d}, 4 \mathrm{f}]$} & val & val & 1.703 & 989 & 7.19 \\
\hline & & AIMP & {$[\mathrm{Kr}, 4 \mathrm{~d}, 4 \mathrm{f}]$} & aug.val & aug.val & 1.706 & 981 & 7.11 \\
\hline & & $\mathrm{AE}$ & $\cdots$ & $\cdots$ & ae & 1.710 & 990 & 7.03 \\
\hline \multirow[t]{5}{*}{$\mathrm{NiO}$} & ${ }^{3} \Sigma^{-}$ & AIMP & {$[\mathrm{Mg}]$} & val & val & 1.595 & 997 & 3.55 \\
\hline & & AIMP & {$[\mathrm{Mg}]$} & aug.val & aug.val & 1.597 & 995 & 3.51 \\
\hline & & AIMP & {$[\mathrm{Ne}]$} & val & val & 1.601 & 995 & 3.48 \\
\hline & & AIMP & {$[\mathrm{Ne}]$} & aug.val & aug.val & 1.601 & 993 & 3.45 \\
\hline & & $\mathrm{AE}$ & $\cdots$ & $\cdots$ & ae & 1.599 & 1010 & 3.49 \\
\hline \multirow[t]{5}{*}{$\mathrm{PdO}$} & ${ }^{3} \Pi$ & AIMP & {$[\mathrm{Zn}]$} & val & val & 1.842 & 519 & 2.90 \\
\hline & & AIMP & {$[\mathrm{Zn}]$} & aug.val & aug.val & 1.829 & 559 & 2.89 \\
\hline & & AIMP & {$[\mathrm{Ar}, 3 \mathrm{~d}]$} & val & val & 1.856 & 461 & 2.85 \\
\hline & & AIMP & {$[\mathrm{Ar}, 3 \mathrm{~d}]$} & aug.val & aug.val & 1.834 & 543 & 2.86 \\
\hline & & $\mathrm{AE}$ & $\cdots$ & $\cdots$ & ae & 1.836 & 542 & 2.80 \\
\hline \multirow[t]{5}{*}{$\mathrm{PtO}$} & ${ }^{3} \Sigma^{-a}$ & AIMP & {$[\mathrm{Cd}, 4 \mathrm{f}]$} & val & val & 1.792 & 1027 & 2.88 \\
\hline & & AIMP & {$[\mathrm{Cd}, 4 \mathrm{f}]$} & aug.val & aug.val & 1.799 & 1020 & 2.78 \\
\hline & & AIMP & {$[\mathrm{Kr}, 4 \mathrm{~d}, 4 \mathrm{f}]$} & val & val & 1.797 & 1024 & 2.82 \\
\hline & & AIMP & {$[\mathrm{Kr}, 4 \mathrm{~d}, 4 \mathrm{f}]$} & aug.val & aug.val & 1.803 & 1014 & 2.73 \\
\hline & & $\mathrm{AE}$ & $\ldots$ & $\cdots$ & $a e$ & 1.790 & 1039 & 2.90 \\
\hline
\end{tabular}

${ }^{\mathrm{a}} \mathrm{ACPF}$ calculations with reference spaces obtained from distributing six electrons in the $\pi$ and $\pi^{\prime}$ orbitals.

partial negative charge; for a proper description of the electron affinity at least one $\mathrm{f}$ polarization function is required. ${ }^{18}$

Somewhat larger errors in calculated spectroscopic parameters are found for the group 10 monoxides, in accord with the results of other theoretical studies. ${ }^{17,19}$ This is not too astonishing as large polarization basis sets are required to account for the differential electronic correlation in the late TM. $^{20,21}$ Furthermore, spin-orbit interaction has a nonnegligible influence on the spectroscopic parameters in the heavier compounds.

For $\mathrm{NiO}$ we underestimate the equilibrium distance by $0.03 \AA$, the vibrational frequency is overestimated by about $160 \mathrm{~cm}^{-1}$ and we retain $88 \%$ of the experimental dissociation energy. Dolg et al. ${ }^{17}$ get a similar deviation for the equilibrium distance, perfect agreement for the vibrational frequency but they retain only $60 \%$ of the experimental dissociation energy. Multireference configuration interaction calculations by Bauschlicher et al. ${ }^{19}$ overestimate the equilibrium distance by $0.04 \AA$ and underestimate the frequency by $140 \mathrm{~cm}^{-1}$, while the single-reference results deviate considerably from experiment. It appears thus that both, large basis sets and a multireference treatment is required for $\mathrm{NiO}$.
For $\mathrm{PdO}$, to our knowledge, no reliable experimental spectroscopic parameters have been determined. Its dissociation energy, from which we retain $77 \%$, was derived from thermochemical data. ${ }^{22}$ The only other theoretical study, which we know of, was performed by Bauschlicher et al. ${ }^{19}$ These authors did not include any kinematic relativistic effects, however. It is thus not astonishing that their results differ considerably from ours.

Even less is known about the properties of the heaviest homolog PtO. For a long time its ground state was erroneously identified as a state of ${ }^{1} \Sigma^{+}$symmetry. $^{22-24}$ In 1983 Sassenberg et al. ${ }^{25}$ concluded from their experimental spectral data that the lowest $\Omega=0^{+}$and 1 states together correspond to a spin-orbit split ${ }^{3} \Sigma^{-}$state. They determined vibrational frequencies of 851 and $832 \mathrm{~cm}^{-1}$ for the $0^{+}$and 1 states, respectively. Our calculated values of 1014 or 1039 $\mathrm{cm}^{-1}$ at the AIMP or AE level of calculation, respectively, are substantially higher. However, as apparent from the substantial second-order splitting between the fine-structure components of the ${ }^{3} \Sigma^{-}$state, spin-orbit effects have a considerable influence on the spectroscopic parameters of $\mathrm{PtO}$ in this state. Although little is known about the excited elec- 
TABLE V. Spectroscopic constants of group 5 and 10 transition metal monoxides. Spin-free relativistic calculations from our work, other theoretical and experimental results.

\begin{tabular}{|c|c|c|c|c|c|c|c|}
\hline Molecule & State & Method & Level & Reference & $R_{e}[\AA]$ & $\omega_{e}\left[\mathrm{~cm}^{-1}\right]$ & $D_{e}[\mathrm{eV}]$ \\
\hline \multirow[t]{6}{*}{ VO } & \multirow[t]{6}{*}{${ }^{4} \Sigma^{-}$} & NP-AIMP ${ }^{a}$ & $\mathrm{MCPF}$ & this work & 1.586 & 1008 & 6.09 \\
\hline & & $N P-A E^{b}$ & $\mathrm{MCPF}$ & this work & 1.588 & 1003 & 6.06 \\
\hline & & $\mathrm{AE}+\mathrm{R}^{\mathrm{c}}$ & $\mathrm{CPF}$ & 15 & 1.604 & 969 & $5.68^{\mathrm{d}}$ \\
\hline & & $\mathrm{PP}^{\mathrm{e}}$, nonrel. & $\mathrm{CI}(\mathrm{SD})+\mathrm{Q}^{\mathrm{f}}$ & 17 & 1.578 & 890 & 5.32 \\
\hline & & Experiment & & 22 & 1.589 & 1011 & $6.41^{\mathrm{d}}$ \\
\hline & & Experiment & & 26 & $\cdots$ & 1011 & $6.44 \pm 0.20$ \\
\hline \multirow[t]{6}{*}{$\mathrm{NbO}$} & \multirow[t]{6}{*}{${ }^{4} \Sigma^{-}$} & NP-AIMP & $\mathrm{MCPF}$ & this work & 1.680 & 1022 & 7.21 \\
\hline & & $N P-A E^{b}$ & $\mathrm{MCPF}$ & this work & 1.676 & 1022 & 7.23 \\
\hline & & $\mathrm{RECP}^{\mathrm{g}}$ & MCPF & 16 & 1.697 & 977 & 6.83 \\
\hline & & $\mathrm{QRPP}^{\mathrm{h}}$ & $\mathrm{ACPF}$ & 13 & 1.675 & 1033 & 6.91 \\
\hline & & Experiment & & 22 & 1.691 & 989 & $7.8^{\mathrm{d}}$ \\
\hline & & Experiment & & 26 & $\cdots$ & 989 & $7.93 \pm 0.26$ \\
\hline \multirow[t]{7}{*}{$\mathrm{TaO}$} & \multirow[t]{3}{*}{${ }^{4} \Sigma^{-}$} & NP-AIMP & $\mathrm{MCPF}$ & this work & 1.706 & 981 & 7.11 \\
\hline & & $N P-A E^{b}$ & MCPF & this work & 1.710 & 990 & 7.03 \\
\hline & & $\mathrm{QRPP}^{\mathrm{j}}$ & $\mathrm{ACPF}$ & 13 & 1.701 & 1004 & 6.91 \\
\hline & \multirow[t]{4}{*}{${ }^{2} \Delta$} & NP-AIMP & $\mathrm{MCPF}$ & this work & 1.689 & 1026 & 7.53 \\
\hline & & $\mathrm{QRPP}^{\mathrm{j}}$ & $\mathrm{ACPF}$ & 13 & 1.691 & 1023 & 7.67 \\
\hline & & Experiment & & 22 & 1.686 & 1030 & $8.2^{\mathrm{d}}$ \\
\hline & & Experiment & & 26 & $\ldots$ & 1030 & $8.24 \pm 0.13$ \\
\hline \multirow[t]{8}{*}{$\mathrm{NiO}$} & \multirow[t]{8}{*}{${ }^{3} \Sigma^{-}$} & NP-AIMP & $\mathrm{MCPF}$ & this work & 1.601 & 993 & 3.45 \\
\hline & & $N P-A E^{b}$ & $\mathrm{MCPF}$ & this work & 1.599 & 1010 & 3.49 \\
\hline & & $\mathrm{PP}^{\mathrm{e}}$, nonrel. & $\mathrm{CI}(\mathrm{SD})+\mathrm{Q}^{\mathrm{f}}$ & 17 & 1.591 & 848 & 2.33 \\
\hline & & $\mathrm{AE}$, nonrel. & $\mathrm{CI}(\mathrm{SD})+\mathrm{Q}^{\mathrm{f}}$ & 19 & 1.50 & 510 & $\ldots$ \\
\hline & & $\mathrm{AE}$, nonrel. & $\operatorname{MRCI}(\mathrm{SD})+\mathrm{Q}^{\mathrm{i}}$ & 19 & 1.67 & 690 & $\cdots$ \\
\hline & & Experiment & & 22 & $\ldots$ & $\cdots$ & $3.87^{\mathrm{d}}$ \\
\hline & & Experiment & & 26 & $\ldots$ & 838 & $3.91 \pm 0.18$ \\
\hline & & Experiment & & 27 & 1.627 & 839 & 3.81 \\
\hline \multirow[t]{8}{*}{$\mathrm{PdO}$} & \multirow[t]{3}{*}{${ }^{3} \Pi$} & NP-AIMP ${ }^{a}$ & $\mathrm{MCPF}$ & this work & 1.834 & 543 & $2.86^{\mathrm{j}}$ \\
\hline & & $N P-A E^{b}$ & $\mathrm{MCPF}$ & this work & 1.836 & 542 & $2.80^{\mathrm{j}}$ \\
\hline & & $\mathrm{AE}$, nonrel. & $\mathrm{CI}(\mathrm{SD})+\mathrm{Q}^{\mathrm{f}}$ & 19 & 1.95 & 480 & $\ldots$ \\
\hline & \multirow[t]{5}{*}{${ }^{3} \Sigma^{-}$} & NP-AIMPa & $\mathrm{MCPF}$ & this work & 1.808 & 636 & 2.15 \\
\hline & & AE, nonrel. & $\mathrm{CI}(\mathrm{SD})+\mathrm{Q}^{\mathrm{f}}$ & 19 & 1.70 & 380 & $\cdots$ \\
\hline & & $\mathrm{AE}$, nonrel. & $\operatorname{MRCI}(\mathrm{SD})^{\mathrm{k}}$ & 19 & 1.95 & 470 & $\ldots$ \\
\hline & & Experiment & & 22 & $\ldots$ & $\ldots$ & $2.87^{\mathrm{d}}$ \\
\hline & & Experiment & & 26 & $\cdots$ & $810^{1}$ & $\cdots$ \\
\hline \multirow[t]{4}{*}{$\mathrm{PtO}$} & \multirow[t]{4}{*}{${ }^{3} \Sigma^{-}$} & NP-AIMP ${ }^{a}$ & $\mathrm{ACPF}$ & this work & 1.803 & 1014 & 2.73 \\
\hline & & $N P-A E^{b}$ & $\mathrm{ACPF}$ & this work & 1.790 & 1039 & 2.90 \\
\hline & & Experiment & & 25 & $\ldots$ & $851^{\mathrm{m}}$ & $\ldots$ \\
\hline & & Experiment & & 25 & $\ldots$ & $832^{\mathrm{n}}$ & $\ldots$ \\
\hline
\end{tabular}

${ }^{\text {a Spin-free relativistic small-core NP-AIMP calculation using the intermediate basis sets of type aug.val for the }}$ representations of the exchange and no-pair operators.

${ }^{\mathrm{b}}$ Spin-free relativistic NP-AE calculation.

${ }^{c}$ Mass-velocity and Darwin contributions have been included using first-order perturbation theory.

${ }^{\mathrm{d}}$ A dissociation energy $D_{0}$ is given.

${ }^{e}$ Energy-adjusted pseudopotential method, SEFIT results are given for the $R_{e}$ and $\omega_{e} ; D_{e}$ is taken from MEFIT calculations.

${ }^{\mathrm{f}} \mathrm{CI}$ calculations with single and double excitations including Davidson correction.

${ }^{g}$ Relativistic effective core potential method.

${ }^{\mathrm{h}}$ Quasirelativistic energy-adjusted pseudopotential method.

${ }^{i} \mathrm{MRCI}$ calculations with single and double excitations including Davidson correction.

${ }^{\mathrm{j}}$ Dissociation to the $\mathrm{s}^{1} \mathrm{~d}^{9}$ excited state of Pd.

${ }^{\mathrm{k}} \mathrm{MRCI}$ calculations with single and double excitations.

'Estimated value according to the authors' note.

${ }^{\mathrm{m}}$ Harmonic vibrational frequency of the $\Omega=0^{+}$state

${ }^{\mathrm{n}}$ Harmonic vibrational frequency of the $\Omega=1$ state.

tronic states of $\mathrm{PtO}$, it can be deduced from the spectra of the lighter homologs $\mathrm{NiO}$ and $\mathrm{PdO}$ that a low-lying ${ }^{3} \Pi$-state should exist in $\mathrm{PtO}$ which can interact with the ground state components via spin-orbit coupling.

\section{CONCLUSIONS}

In this work, we complement our previous study on relativistic no-pair $a b$ initio model potentials and valence basis 
sets for the transition elements $\mathrm{Sc}-\mathrm{Hg}$. AIMPs and valence basis sets corresponding to valence spaces comprising the $n \mathrm{~s},(n-1) \mathrm{d},(n-1) \mathrm{p}$, and $(n-1) \mathrm{s}$ shells are provided. Atomic properties obtained from AIMP Hartree-Fock calculations exhibit very good agreement with corresponding allelectron HF calculations. Excellent agreement between AIMP and AE results is obtained at the HF and the correlated levels for equilibrium distances and vibrational frequencies. As expected, the explicit inclusion of the $(n-1) \mathrm{s}$ semicore into the valence space turns out to be more important for early than for late transition metal monoxides. Relativistic operators are sufficiently well represented in the valence basis. Augmenting the resolution of the identity for the exchange operator, on the other hand, improves dissociation energies, in particular of early TM monoxides, bringing them into close agreement with the AE results. In this case it is possible to reduce the third-row TM $\mathrm{f}$ valence basis sets without loss of accuracy which implies considerable savings in computational resources. For the group 5 TM monoxides our results agree very well with experiment, while for the group 10 TM monoxides inclusion of additional static and dynamic correlation effects and spin-orbit coupling seems to be indicated.

\section{ACKNOWLEDGMENTS}

Financial support by the German Research Council (DFG Priority Project "Relativistic Effects," Ma 1051/2-2) and MEC, Spain, (PB95-0201) is gratefully acknowledged. Further, we express our gratitude to the European Science Foundation (REHE-Programme), the Spanish and German Academic Exchange Services (Acciones Integradas, contract number HA1997-0097, and DAAD, contract number 314AI-e-dr) for traveling funds.

${ }^{1}$ F. Rakowitz, C. M. Marian, L. Seijo, and U. Wahlgren, J. Chem. Phys. 110, 3678 (1999).

${ }^{2}$ S. Huzinaga, L. Seijo, Z. Barandiarán, and M. Klobukowski, J. Chem. Phys. 86, 2132 (1987).
${ }^{3}$ See http://www.thch.uni-bonn.de/tc/TCB.download.html for MP parameters, valence and augmentation basis sets.

${ }^{4}$ B. A. Hess, Phys. Rev. A 33, 3742 (1986).

${ }^{5}$ R. Samzow. Ph.D. thesis, University of Bonn, 1991.

${ }^{6}$ MOLECULE-SWEDEN is an electronic structure program package, written by J. Almlöf, M. R. A. Blomberg, L. G. M. Petterson, B. O. Roos, and P. E. M. Siegbahn. Program modifications to include no-pair relativistic integrals by B. A. Hess, C. M. Marian, and U. Wahlgren.

${ }^{7}$ ECPAIMP is an integral program for ECP and AIMP calculations written by L. G. M. Pettersson, L. Seijo, and M. A. Nygren.

${ }^{8}$ MOLCAS version 4. K. Andersson, M. R. A. Blomberg, M. P. Fülscher, G. Karlström, R. Lindh, P.-Å. Malmqvist, P. Neogrády, J. Olsen, B. O. Roos, A. J. Sadlej, M. Schütz, L. Seijo, L. Serrano-Andrés, P. E. M. Siegbahn, and P.-O. Widmark, Lund University, Sweden (1997).

${ }^{9}$ D. P. Chong and S. R. Langhoff, J. Chem. Phys. 84, 5606 (1986).

${ }^{10}$ R. J. Gdanitz and R. Ahlrichs, Chem. Phys. Lett. 143, 413 (1988).

${ }^{11}$ A. J. Merer, Annu. Rev. Phys. Chem. 40, 407 (1989).

${ }^{12}$ J. M. Brom, C. H. Durhamm, and W. Weltner, J. Chem. Phys. 61, 970 (1974).

${ }^{13}$ M. Dolg, H. Stoll, H. Preuss, and R. M. Pitzer, J. Phys. Chem. 97, 5852 (1993).

${ }^{14}$ M. Casarrubios and L. Seijo, J. Chem. Phys. 110, 784 (1999).

${ }^{15}$ C. W. Bauschlicher, Jr. and S. R. Langhoff, J. Chem. Phys. 85, 5936 (1986).

${ }^{16}$ S. R. Langhoff and C. W. Bauschlicher, Jr., J. Chem. Phys. 89, 2160 (1988).

${ }^{17}$ M. Dolg, U. Wedig, H. Stoll, and H. Preuss, J. Chem. Phys. 86, 2123 (1987).

${ }^{18}$ R. A. Kendall, Jr., T. H. Dunning, and R. J. Harrison, J. Chem. Phys. 96, 6796 (1992)

${ }^{19}$ C. W. Bauschlicher, Jr., C. J. Nelin, and P. S. Bagus, J. Chem. Phys. 82, 3265 (1985)

${ }^{20}$ B. H. Botch, Jr., T. H. Dunning, and J. F. Harrison, J. Chem. Phys. 75, 3466 (1981)

${ }^{21}$ C. W. Bauschlicher, Jr. and S. P. Walch, J. Chem. Phys. 76, 1033 (1982).

${ }^{22}$ K. P. Huber and G. Herzberg, Molecular Spectra and Molecular Structure, IV. Constants of Diatomic Molecules (Van Nostrand Reinhold, New York, 1979).

${ }^{23}$ R. Scullman, U. Sassenberg, and C. Nilsson, Can. J. Chem. 53, 1991 (1975).

${ }^{24}$ C. Nilsson, R. Scullman, and N. Mehendalé, J. Mol. Spectrosc. 35, 177 (1970).

${ }^{25}$ U. Sassenberg and R. Scullman, Phys. Scr. 28, 139 (1983).

${ }^{26}$ J. B. Pedley and E. M. Marshall, J. Phys. Chem. Ref. Data 12, 967 (1983).

${ }^{27}$ V. I. Srdanov and D. O. Harris, J. Chem. Phys. 89, 2748 (1988). 